were tracked by way of the Aubrey holes at Stonehenge, might have come to be worshipped as a powerful, though unseen, god. He therefore concludes the book by querying: "Could a distant memory of [the Sun, Moon and lunar nodes] be the origin of the doctrine of the Trinity, the "three in one, the one in three'? I suspect so. In Stonehenge I may well be many roots of our present-day culture."

\section{Microscopic techniques}

Analytical and Quantitative Methods in Microscopy. Edited by G. A. Meek and H. Y. Elder. Pp. 276. (Cambridge University: Cambridge, London and New York, 1977.) Hardback £12; paperback $£ 4.75$.

THIS volume originates in a symposium on principles, applications and shortcomings of some techniques in modern microscopy, organised under the auspices of the Society for Experimental Biology in 1975. It consists of an introduction and twelve chapters. The topics considered are stereology, optical diffraction analysis, quantitative fluorescence microscopy, image analysis, integrating microdensitometry, scanning microinterferometry, STEM, microanalysis of various sorts, and cryoultramicrotomy.

Most of the chapters are written in a clear instructive style which is fairly easy to follow, although some of the authors have lapsed on occasion into the more easily produced review type of text. The editors have done well to cajole a panel of authors of this size into producing a reasonably uniform style and standard of presentation. The chapters seem to have very few statements to which one could take specific objection, although it is difficult for one person to critically encompass accounts of all of these topics. Each of the chapters is at such a level and of

\section{Insect \\ behaviour-modifying chemicals}

Chemical Control of Insect Behaviour. Edited by H. H. Shorey and J. J. McKelvey, Jr. Pp. 414. (WileyInterscience: New York and London, 1977.) $\$ 24.50 ; £ 14.65$.

THIS book is the eighteenth publication in the series Environmental Science and Technology, and deals with a subject that, because of its likely contribution to a more rational use of insecticides, is of particular
No doubt the student who stood me up for Stonehenge will feel confirmed in her choice by these words. Those who would not wish to venture so far should, nevertheless, be grateful to Sir Fred Hoyle for setting out the case for an astronomical interpretation of Stonehenge in such a readable manner.

Jack Meadows is Professor of Astronomy and History of Science at the University of Leicester, UK.

a style as to make it very suitable first reading for a research student or a more senior researcher contemplating working in one of these areas of technique. Such readers will get a helpful mechanistic accounts of the methods concerned. The chapters by $\mathbf{R}$. W. Horne (optical diffraction analysis), D. J. Goldstein (microinterferometry and microdensitometry) and S. Bradbury (quantitative image analysis) are particularly helpful and timely accounts.

Perhaps it should be asked how fair a picture this book gives of microscopy today. Taken together, they describe methodology that finds use at present in a small minority of research projects. The overall picture of microscopy presented in this volume is one that emphasises certain (sometimes quite isolated) specialities. It is for the most part about the iced cakes of microscopy rather than the bread and butter.

Perhaps the ultimate test of a book that is designed for the teaching of a technique is to see if it can tell the researcher whether a particular approach is appropriate for answering the questions he wishes to ask. I am not certain how many of the chapters pass this test, but there are some really excellent helpful accounts of certain microscope technologies in this book. The paperback version, in particular, is fine value.

\section{A. Williams}

M. A. Williams is Reader in Human Biology and Anatomy at the University of Sheffield, UK.

relevance to the study of the environment and the technology of its conservation. All types of behaviour-modifying chemical known to influence insect behaviour by external reception, including pheromones and plant-derived compounds, are covered and their prospects in pest management are discussed.

Publication of the book has arisen from a conference, involving many leading workers in the field, held in May 1975 at Bellagio, Italy, with participants contributing authoritative chapters on the various aspects of the subject. After a short introduction, subsequent chapters are arranged into five sections. The first deals with the nature of sensory responses to behaviour-modifying chemicals with many examples from electrophysiological studies. The next two sections are devoted to behavioural aspects and begin with a detailed account of the various mechanisms by which insects respond to distant odour sources. The fourth section considers the role of diversity in behaviourmodifying chemicals, with the approaches to their use in insect pest managment reviewed at length in the final section. Each chapter has an extensive list of references including many publications from 1975. The book has a comprehensive subject index.

The present high level of research activity in chemical control of insect behaviour may mean that this book, dealing with the subject in such depth, will soon become out of date. Already, for example, the treatment of chemoklinotaxis in chapter 5 has been revised by the author, and in chapter 9 a point is made based on a recently questioned report that the sex pheromone of Musca domestica consists of one compound. Nevertheless, since the authors have, with only a few notable exceptions, been cautious in drawing conclusions from the data presented and are mostly in a position to judge this data critically, the likelihood of serious reappraisal of much of the content is greatly reduced.

As the book is compiled from many individual contributions, apparently without too rigorous editing, there is inevitably some duplication of information, and in some cases the treatment of such data seems to be inconsistent. This often presents the reader, however, with the opportunity to compare the various current approaches to controversial issues. For example, the subject of kairomones is treated in several different ways, and a number of authors make different suggestions for improving definitions of the terms that have been devised to fulfil the requirements of the subject.

The prospects for insect behaviourmodifying chemicals in pest management are considered critically and without the rash claims typical of many earlier writings. In addition, a number of realistic suggestions for new approaches are made although it is asserted that more rapid progress would be made in pest management if priority could be given to fundamental research in certain listed areas.

There is no doubt that this book is to be recommended. It will be of most value to those already having some knowledge of the subject and will provide a stimulating background on which to base new research. In addition, those engaged in deciding priorities for future crop protection research and development will find this book a source of useful information and ideas.

J. A. Pickett

J. A. Pickett is Principal Scientific Officer coordinating chemical aspects of work on behaviourcontrolling chemicals at Rothamsted Experimental Station, Harpenden, $U K$ 